\title{
Use of a Tablet-Based System to Perform Abdominal Ultrasounds in a Field Investigation of Schistosomiasis-Related Morbidity in Western Kenya
}

\author{
Anne Straily, ${ }^{1 \star}$ Alfred O. Malit, ${ }^{2}$ Dollycate Wanja, ${ }^{2}$ Emmy A. Kavere, ${ }^{2}$ Rono Kiplimo, ${ }^{2}$ Rose Aera, ${ }^{2}$ Caroline Momanyi, ${ }^{2}$ \\ Solomon Mwangi, ${ }^{2}$ Sarah Mukire, ${ }^{2}$ Ashley A. Souza, ${ }^{3}$ Ryan E. Wiegand,,${ }^{1,4,5}$ Susan P. Montgomery, ${ }^{1}$ William E. Secor, ${ }^{1}$ and \\ Maurice R. Odiere 2,6 \\ ${ }^{1}$ Parasitic Diseases Branch, Division of Parasitic Diseases and Malaria, Centers for Disease Control and Prevention, Atlanta, Georgia; ${ }^{2}$ Safe Water \\ and AIDS Project, Kisumu, Kenya; ${ }^{3}$ Task Force for Global Health, Atlanta, Georgia; ${ }^{4}$ Swiss Tropical and Public Health Institute, Basel, Switzerland; \\ ${ }^{5}$ University of Basel, Basel, Switzerland; ${ }^{6}$ Centre for Global Health Research, Kenya Medical Research Institute, Kisumu, Kenya
}

\begin{abstract}
Chronic intestinal schistosomiasis can cause severe hepatosplenic disease and is a neglected tropical disease of public health importance in sub-Saharan Africa, including Kenya. Although the goal of control programs is to reduce morbidity, milestones for program performance focus on reductions in prevalence and intensity of infection, rather than actual measures of morbidity. Using ultrasound to measure hepatosplenic disease severity is an accepted method of determining schistosomiasis-related morbidity; however, ultrasound has not historically been considered a fielddeployable tool because of equipment limitations and unavailability of expertise. A point-of-care tablet-based ultrasound system was used to perform abdominal ultrasounds in a field investigation of schistosomiasis-related morbidity in western Kenya; during the study, other pathologies and pregnancies were also identified via ultrasound, and participants referred to care. Recent technological advances may make it more feasible to implement ultrasound as part of a control program and can also offer important benefits to the community.
\end{abstract}

Intestinal schistosomiasis, caused by Schistosoma mansoni, affects an estimated 54 million persons in sub-Saharan Africa, with more than $50 \%$ of resultant morbidity found around the African Great Lakes, including Kenya. ${ }^{1}$ Adult S. mansoni worms reside in the mesenteric venules of infected humans; female worms release eggs that become trapped in the tissues, primarily the liver, where their presence elicits a granulomatous inflammatory reaction that can result in morbidity. Severe morbidity due to intestinal schistosomiasis is characterized by hepatosplenic disease, including periportal fibrosis, and develops over $5-15$ years of infection. ${ }^{2}$ Advanced periportal fibrosis and resultant portal hypertension can lead to development of esophageal varices, which are rapidly fatal when ruptured. Although the WHO's goal for control programs is to reduce morbidity, evaluation measures focus on reductions in prevalence and infection intensity, rather than actual measures of morbidity. ${ }^{3}$ This is largely because of challenges in measuring severe morbidity in the field and because symptoms associated with less severe schistosomiasis morbidity can result from other causes. ${ }^{4}$ Country control programs need morbidity indicators that can be reliably measured, and the WHO has designated identification of these markers as a critical action to achieve elimination of schistosomiasis as a public health problem. $^{5}$ In 2019 , the Morbidity Operational Research for Bilharziasis Implementation Decisions (MORBID) pilot study was launched in an S. mansoni-endemic area of western Kenya to identify better methods to detect morbidity for control programs. Morbidity was assessed by several methods, one of which was ultrasound of the liver and spleen. The full project, including methods and results, will be further described elsewhere (Odiere et al., manuscript in preparation).

Ultrasound is a useful diagnostic modality to directly visualize the liver and spleen. It is noninvasive, relatively simple to

*Address correspondence to Anne Straily, Centers for Disease Control and Prevention, 1600 Clifton Rd. NE, H24-3, Atlanta, GA 30329. E-mail: yzv2@cdc.gov perform, generally has high community acceptance, rapidly provides results, and can distinguish between fibrosis caused by $S$. mansoni infection and other causes of hepatic disease (e.g., cirrhosis or viral hepatitis). ${ }^{6-11}$ However, ultrasound has not been considered a feasible, field-deployable tool for routine use in control programs because of costs and limitations of machines and availability of ultrasonography expertise. Older models of ultrasound machines were heavy, bulky, and required an external power source, which greatly affected transportation needs, personnel, logistics, and costs. For example, during the Schistosomiasis Control Operational Research and Evaluation (SCORE) morbidity studies, the ultrasound machine used in Kenya was a refurbished Aloka SSD-900 portable model† (Aloka, Tokyo, Japan). ${ }^{12}$ It weighed approximately $14.5 \mathrm{~kg}$ and required a generator and gasoline for field use. That model could not store images electronically, so they were printed on special thermal paper after each examination for further study and review; however, the images faded quickly, making them nearly impossible to evaluate retrospectively (W. E. Secor, personal communication). When the Aloka unit was purchased in 2011 , it cost $\$ 11,000$ US dollars (USD) with an additional $\$ 800$ USD for the printer (not including cost of shipping the unit to Kenya). Updated clinical machines with equivalent features now cost anywhere from $\$ 22,000$ to $\$ 36,000$, $\neq$ putting them financially out of reach of many country control programs or field studies.

Recently, tablet-based ultrasound systems consisting of a transducer connected to a compatible smart device have become available. We evaluated whether such a system would be useful to assess hepatosplenic disease related to intestinal schistosomiasis in a field study setting. We used the

†Use of trade names and commercial sources is for identification only and does not constitute endorsement by the Centers for Disease Control and Prevention or the US Department of Health and Human Services.

†Price as of $8 / 21 / 2020$ when quote was obtained. 

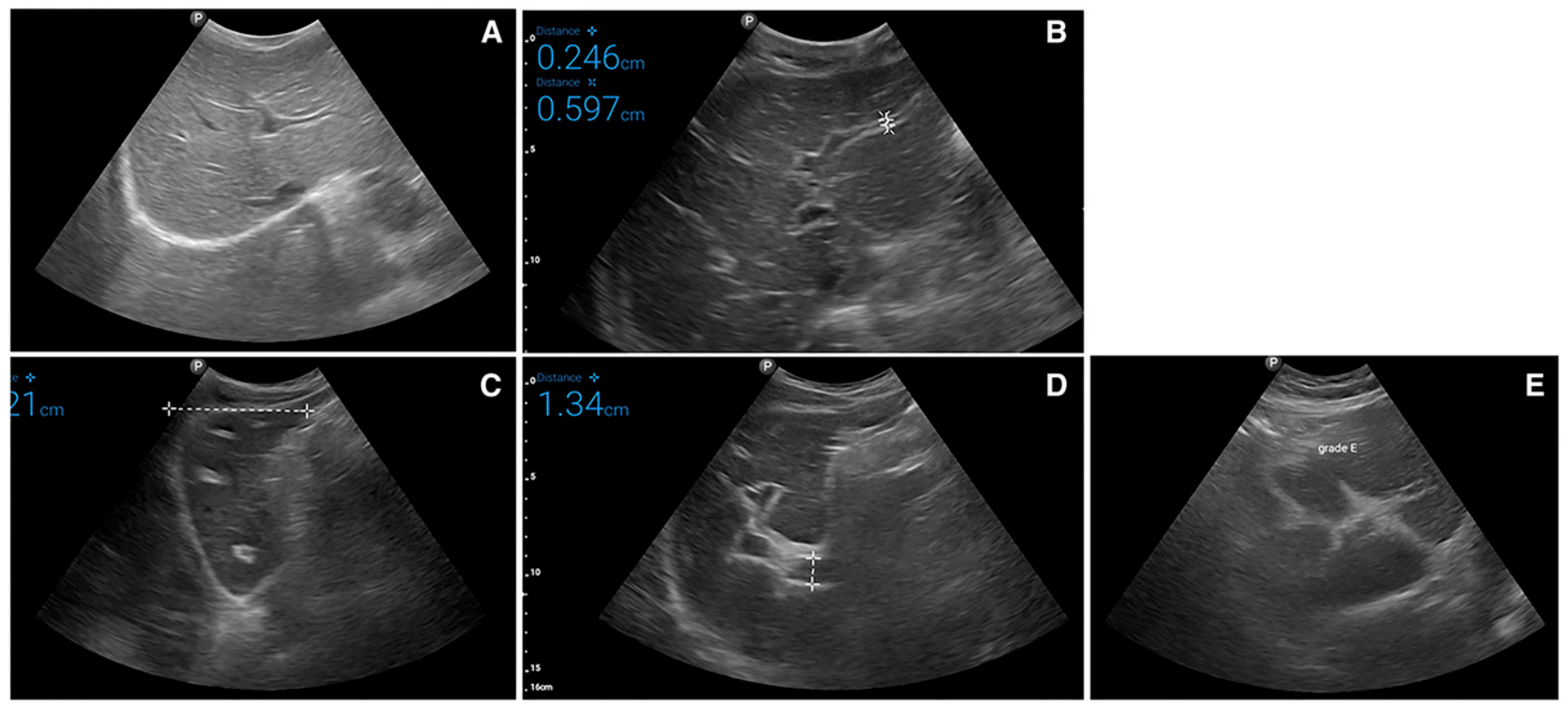

FIGURE 1. Examples of schistosomiasis-related ultrasound liver texture patterns, as defined by the WHO's Niamey protocol, ${ }^{13}$ observed during the Morbidity Operational Research for Bilharziasis Implementation Decisions study. (A) normal liver parenchyma; (B) "starry sky"; (C) "rings" (seen here) and "pipe stems," which correspond to the "rings" seen in a scan perpendicular to the one where pipe stems are seen; (D) highly echogenic "ruff" seen around the portal bifurcation and main stem; (E) highly echogenic "patches" extending from the main portal vein and branches into the parenchyma. This figure appears in color at www.ajtmh.org.

Lumify system by Philips Medical Imaging† (Bothell, WA), consisting of the $\mathrm{C} 5-2 \mathrm{MHz}$ broadband curved array transducer and compatible Android tablets with the Lumify app installed. The Lumify system is ideal for field applications: it consists simply of the transducer and tablet and is therefore light, easily transportable, and does not require an external power source to perform the ultrasound. The display has excellent resolution, comparable to a clinical ultrasound machine. High-definition images and short videos can be saved on the tablet for later review. The transducers can be leased for a period of time, which reduces costs. For the Kenya MORBID pilot study, four broadband curved array transducers were leased and four compatible tablets were purchased.

The MORBID sonographers were recent graduates of a medical imaging training program in Nairobi, Kenya. They were proficient in conducting ultrasound examinations but needed additional training to identify different patterns of schistosomiasis-specific hepatosplenic disease as standardized by the WHO. ${ }^{13}$ In June 2019, the team traveled to the Kabatereine Schistosomiasis Research Centre at Bugoigo field station in Buliisa district, Uganda, to undergo an intensive 3-day training in schistosomiasis-specific ultrasonography based on the Niamey protocol. ${ }^{13}$ Bugoigo field station is situated close to Lake Albert where intestinal schistosomiasis is endemic and morbidity is common, enabling the trainees to observe ultrasound patterns associated with advanced schistosomiasis.

The MORBID study protocol was reviewed and approved by the Institutional Review Board of Maseno University, Kisumu, Kenya (Protocol \#MSU/DRPI/MUERC/00675/19), and by the Office of the Associate Director for Science in the Center for Global Health, Centers for Disease Control and Prevention (CDC) investigators were considered to be nonengaged with human subjects. The MORBID study was conducted in 45 villages, with approximately 200 residents recruited from each village. In total, 6,483 participants received an abdominal ultrasound. Between 20 and 25 participants were examined each day. Images were viewed in real time and saved electronically to the tablet. Each afternoon, upon returning from the field, images were downloaded and saved to an external hard drive and the tablets recharged. Images were reviewed and scored for schistosomiasis-related hepatosplenic pathology according to standardized guidelines (Figure 1). ${ }^{13}$ Study morbidity findings will be summarized elsewhere (Odiere et al., manuscript in preparation).

Although the study's objective was to identify hepatosplenic pathologies associated with schistosomiasis, each participant was given a complete abdominal ultrasound examination. In addition, non-schistosomiasis-associated pathologies were thereby identified, including uterine fibroids, polycystic kidney disease, hydronephrosis, prostatic hypertrophy, fatty liver, and liver cysts. Many areas of Kenya lack this type of advanced imaging capability, and these pathologies may otherwise have gone undiagnosed. Where further evaluation by a physician was needed, the sonographer generated a report and worked with local community health volunteers to refer the participant to a local hospital. For example, an adult male participant with severe lower limb edema was found to have advanced prostatic hyperplasia causing venous occlusion. He reported that he had been variously treated for other potential causes of his lower limb edema but had never received any imaging studies. Twenty-one pregnancies were diagnosed in women, some of whom were not aware that they were pregnant; the sonographers were able to determine gestational age, expected delivery date, and fetal sex. Participants taking medication for an abdominopelvic 
condition were able to receive feedback, for example, whether the condition was resolving, and were provided a report to take to their physician. One participant was identified with situs inversus, a rare congenital condition whereby the major visceral organs are reversed from their normal positions; this individual displayed no symptoms or complications.

A tablet-based ultrasound system offers several advantages over a standard ultrasound machine for assessing schistosomiasis morbidity. The battery life was sufficient to perform 25 ultrasounds per day (the number of abdominal ultrasounds that one sonographer could comfortably perform) and did not require recharging while in the field. The images were stored electronically on the device, negating the need to print the images at the time of the examination as was carried out in previous studies, and the device's memory was sufficient to store the images for all study participants examined that day. The tablet and transducer were very easy to transport to, from, and within field sites. This system is also significantly more affordable than a standard ultrasound machine. To rent four Lumify transducers for 6 months ( $\$ 1,200$ USD) and purchase four tablets $(\$ 15,000$ Kenyan Shillings without Value Added Tax or about $\$ 140$ USD each) was roughly half the cost of purchasing the Aloka in 2011 for the SCORE studies. The Lumify system includes the transducer, app, and access to technical support and telemedicine capabilities, but not a smart device. A comparable standard ultrasound machine retails for \$22,000-36,000 USD $\ddagger$ and still requires an external power source to perform the examination. Another limitation of performing ultrasound in the field is the expertise needed to conduct the examination and interpret the images. We found that contracting recent graduates of a medical imaging training program and providing additional training necessary to identify schistosomiasis-specific pathologic changes was an effective solution for meeting this need. Another potential strategy is to develop focused point-ofcare ultrasound applications which target only specific findings necessary for diagnosis and treatment decisions and provide training to clinicians already working in affected communities. $^{14}$

Measuring severity of hepatosplenic disease is an accepted method of determining schistosomiasis-related morbidity. Recent advances in ultrasound technology which increase portability and decrease costs make it more feasible to incorporate this technology into a country control program and can also offer significant ancillary benefits to the community, such as the opportunity to identify other pathologies and refer people to care, as described here. Integrating ultrasound into control programs will help countries both reduce schistosomiasis morbidity and achieve the WHO's vision of universal health coverage by providing communities with sustainable access to advanced health services. ${ }^{5}$

Received September 10, 2020. Accepted for publication November 9 , 2020.

Published online January 11, 2021.

Financial support: This work received financial support from the Coalition for Operational Research on Neglected Tropical Diseases (COR-NTD), which is funded at The Task Force for Global Health primarily by the Bill \& Melinda Gates Foundation, by U.K. Aid from the British government, and by the U.S. Agency for
International Development through its Neglected Tropical Diseases Program.

Authors' addresses: Anne Straily, Susan P. Montgomery and William E. Secor, Division of Parasitic Diseases and Malaria, Parasitic Diseases Branch, Centers for Disease Control and Prevention, Atlanta, GA, E-mails: yzv2@cdc.gov, zqu6@cdc.gov, and was4@cdc.gov. Alfred Omandi Malit, Dollycate Wanja, Emmy Kavere Awino, Rono Kiplimo, Rose Aera, Caroline Momanyi, Solomon Mwangi, and Sarah Mukire, Safe Water and AIDS Project, Kisumu, Kenya, E-mails: alfredmalit65@gmail.com, wanjadollycate@gmail.com, awinoemmy01@ gmail.com, kiplimorono52@gmail.com, roseaera2014@gmail.com, carolinemomanyi1@gmail.com, solomwas94@gmail.com, and mukire01@gmail.com. Ashley A. Souza, Task Force for Global Health, Atlanta, GA, E-mail: asouza@taskforce.org. Ryan E. Wiegand, Division of Parasitic Diseases and Malaria, Parasitic Diseases Branch, Centers for Disease Control and Prevention, Atlanta, GA, Swiss Tropical and Public Health Institute, Basel, Switzerland, and University of Basel, Basel, Switzerland, E-mail: fwk2@cdc.gov. Maurice R. Odiere, Safe Water and AIDS Project, Kisumu, Kenya, and Centre for Global Health Research, Kenya Medical Research Institute, Kisumu, Kenya, E-mail: mauriceodiere@ gmail.com.

This is an open-access article distributed under the terms of the Creative Commons Attribution (CC-BY) License, which permits unrestricted use, distribution, and reproduction in any medium, provided the original author and source are credited.

\section{REFERENCES}

1. van der Werf MJ, de Vlas SJ, Brooker S, Looman CW, Nagelkerke NJ, Habbema JD, Engels D, 2003. Quantification of clinical morbidity associated with schistosome infection in subSaharan Africa. Acta Trop 86: 125-139.

2. Colley DG, Bustinduy AL, Secor WE, King CH, 2014. Human schistosomiasis. Lancet 383: 2253-2264.

3. World Health Organization, 2013. Schistosomiasis Progress Report 2001-2011, Strategic Plan 2012-2020. Geneva, Switzerland: WHO.

4. King $\mathrm{CH}$, Dangerfield-Cha $\mathrm{M}, 2008$. The unacknowledged impact of chronic schistosomiasis. Chronic Illn 4: 65-79.

5. World Health Organization, 2020. Ending the Neglect to Attain the Sustainable Development Goals: a Road Map for Neglected Tropical Diseases 2021-2030. Geneva, Switzerland: WHO.

6. Homeida M, Abdel-Gadir AF, Cheever AW, Bennett JL, Arbab BM, Ibrahium SZ, Abdel-Salam IM, Dafalla AA, Nash TE, 1988. Diagnosis of pathologically confirmed Symmers' periportal fibrosis by ultrasonography: a prospective blinded study. $A m \mathrm{~J}$ Trop Med Hyg 38: 86-91.

7. Homeida M, Ahmed S, Dafalla A, Suliman S, Eltom I, Nash T, Bennett JL, 1988. Morbidity associated with Schistosoma mansoni infection as determined by ultrasound: a study in Gezira, Sudan. Am J Trop Med Hyg 39: 196-201.

8. Kariuki HC, Mbugua G, Magak P, Bailey JA, Muchiri EM, Thiongo FW, King CH, Butterworth AE, Ouma JH, Blanton RE, 2001. Prevalence and familial aggregation of schistosomal liver morbidity in Kenya: evaluation by new ultrasound criteria. $J$ Infect Dis 183: 960-966.

9. Fataar S, Bassiony H, Satyanath S, Vassileva J, Hanna RM, 1984. Characteristic sonographic features of schistosomal periportal fibrosis. AJR Am J Roentgenol 143: 69-71.

10. Cerri GG, Alves VA, Magalhães A, 1984. Hepatosplenic Schistosomiasis mansoni: ultrasound manifestations. Radiology 153: 777-780.

11. Abdel-Wahab MF, Esmat G, Milad M, Abdel-Razek S, Strickland GT, 1989. Characteristic sonographic pattern of schistosomal hepatic fibrosis. Am J Trop Med Hyg 40: 72-76.

12. Sircar AD, Mwinzi PNM, Onkanga IO, Wiegand RE, Montgomery SP, Secor WE, 2018. Schistosoma mansoni mass drug administration regimens and their effect on morbidity among schoolchildren over a 5-year period-Kenya, 2010-2015. Am J Trop Med Hyg 99: 362-369.

13. World Health Organization, 2000. Ultrasound in Schistosomiasis- $A$ Practical Guide to the Standardized Use of Ultrasonography for 
the Assessment of Schistosomiasis-Related Morbidity. Second International Workshop. October 22-26, 1996. Niamey, Niger. Available at: https://www.who.int/schistosomiasis/resources/ tdr_str_sch_00.1/en/. Accessed May 29, 2020.
14. Bélard S, Tamarozzi F, Bustinduy AL, Wallrauch C, Grobusch MP, Kuhn W, Brunetti E, Joekes E, Heller T, 2016. Point-of-care ultrasound assessment of tropical infectious diseases-a review of applications and perspectives. Am J Trop Med Hyg 94: 8-21. 